\title{
FABLET Dominique (coord.). Les professionnels de l'intervention socio-éducative. Modèles de référence et analyses de pratiques
}

Paris : L'Harmattan, 2007. - 216 p.

\section{Catherine Sellenet}

\section{(2) OpenEdition}

\section{Journals}

Édition électronique

URL : http://journals.openedition.org/rfp/1183

DOI : $10.4000 / \mathrm{rfp} .1183$

ISSN : 2105-2913

Éditeur

ENS Éditions

\section{Édition imprimée}

Date de publication : 1 octobre 2008

Pagination : 140-141

ISBN : 978-2-7342-1143-3

ISSN : 0556-7807

\section{Référence électronique}

Catherine Sellenet, « FABLET Dominique (coord.). Les professionnels de l'intervention socio-éducative. Modèles de référence et analyses de pratiques », Revue française de pédagogie [En ligne], 165 | octobredécembre 2008, mis en ligne le 01 octobre 2010, consulté le 25 septembre 2020. URL : http:// journals.openedition.org/rfp/1183; DOI : https://doi.org/10.4000/rfp.1183

Ce document a été généré automatiquement le 25 septembre 2020.

(c) tous droits réservés 


\section{FABLET Dominique (coord.). Les professionnels de l'intervention socio-éducative. Modèles de référence et analyses de pratiques}

Paris : L'Harmattan, 2007. - 216 p.

\section{Catherine Sellenet}

\section{RÉFÉRENCE}

FABLET Dominique (coord.). Les professionnels de l'intervention socio-éducative.

Modèles de référence et analyses de pratiques. Paris : L'Harmattan, 2007. - 216 p.

1 Le livre coordonné par Dominique Fablet, Les professionnels de l'intervention socioéducative, s'inscrit dans une collection centrée sur l'éducation familiale, définie comme l'étude des pratiques éducatives intrafamiliales et des interventions professionnelles mises en œuvre pour aider les parents. Poursuivant son objectif de diffusion des travaux articulant théories et actions sur le terrain, Dominique Fablet nous offre un ouvrage organisé en quatre parties. Dans la première partie intitulée Professionnalités, Dominique Fablet aide le lecteur à se repérer dans la nébuleuse des «travailleurs sociaux». Son approche historique dégage des temps dans la transformation du paysage institutionnel, dans la création des diplômes et rend intelligible la variété des positionnements possibles des professionnels. Les principales évolutions des pratiques d'intervention étant posées, la contribution des auteurs cerne, plus particulièrement, l'intervention des éducateurs en éducation spécialisée (D. Fablet), celle des éducateurs de jeunes enfants (A.-M. Doucet-Dahlgren), celle des TISF à domicile en protection de l'enfance (B. Tillard). Les trois articles ont en commun de montrer les remaniements des savoirs, le recentrage des interventions en fonction des mutations de la société et des conceptions de l'enfance et de la famille. La diversification des modèles 
d'intervention mais aussi les zones de tensions entre savoirs "anciens » et savoirs "modernes", savoirs experts et savoirs profanes apparaissent clairement. Deux questions d'actualité sont également largement discutées: la question de l'accompagnement et du travail avec les familles, la question de l'évaluation des interventions.

2 La seconde partie, intitulée Approches diversifiées, pluralité des références, décrypte trois approches théoriques soutenant l'intervention : l'approche clinique (M. Boutanquoi, J.P Minary), la pédagogie sociale (H. Milova), l'empowerment (G. Boutin). L'intérêt de ces trois contributions est manifeste dans la distance critique prise avec certains concepts, utilisés souvent sans discernement. Les auteurs pointent les points forts et les limites de notions, largement médiatisées, comme la notion de "bonnes pratiques", les notions d'émancipation et d'épanouissement, la notion de responsabilisation du sujet. D'une contribution à l'autre, un même fil directeur soutient la lecture, à savoir une clarification des notions employées et une interrogation sur leur impact vis-à-vis des pratiques des professionnels. Des notions, qui souvent émaillent les discours sans véritable ossature, sont ainsi dialectisées et réinterrogées au regard de la pratique.

3 La troisième partie se centre sur Familles et usagers: une nouvelle donne? Le titre, explicite, interroge les rapports de pouvoir entre les usagers et les institutions. Les trois contributions proposées (G. Gayet, J.-M. Lesain-Delabarre, L. Barbe) définissent et questionnent les notions d'accompagnement, de dépendance des familles, les représentations à l'œuvre, l'articulation entre les droits des usagers et les pratiques identifiées. Finalement, les pratiques ont-elles bougé ? Assistons-nous aux prémisses de nouvelles alliances entre les professionnels et les parents, ou les mots employés ne sont-ils que les nouveaux habits de pratiques anciennes?

4 Sans conclure, mais en maintenant le questionnement ouvert, la quatrième partie s'ouvre sur l'évaluation des pratiques professionnelles et la nécessité de potentialiser les recherches-actions. Analyser les pratiques professionnelles par la recherche, tel reste l'objectif central défendu par M. Corbillon pour le milieu ouvert, par D. Fablet pour les recherches socio-cliniques et par P. Breugnot pour une lecture de ce que l'on appelle aujourd'hui des pratiques innovantes.

5 La lecture de ce livre présente un double intérêt : celui de créer des ponts entre théorie et action, entre chercheurs et praticiens; mais aussi celui de définir, dialectiser des termes, supports de pensées et de pratiques. L'ancrage des contributions à partir d'observations et de recherches faites sur le terrain, offre aux professionnels des expériences incarnées, concrètes, permettant un juste équilibre entre théorie et pratique. Même si le champ professionnel de l'intervention socio-éducative n'est pas homogène, on retrouve dans la variété des expériences évoquées une même problématisation, que l'on pourrait résumer en ces termes: comment intervenir aujourd'hui dans la sphère familiale, comment aider voire suppléer les parents sans empiéter sur leurs droits, comment soutenir sans rendre dépendant, comment transposer des concepts novateurs en pratiques «bientraitantes", puisque ce terme même a fait son apparition dans le vocabulaire ? La richesse de l'ouvrage provient de la confrontation sans concession des approches, des lieux, des postures. Au-delà, le lecteur y trouvera des indications précieuses sur le jeu entre les acteurs et sur les perceptions des parents qui ne sont jamais absentes des contributions. 


\section{AUTEURS}

CATHERINE SELLENET

Université de Nantes 